\title{
Enhanced antitumor effects of a dendritic cell vaccine transfected with gastric cancer cell total RNA carrying the 4-1BBL gene in vitro
}

\author{
ZHENCHUAN SONG, CHENJUN GUO, YONG LI, BIBO TAN, LIQIAO FAN and JIANWEI XIAO
}

Department of Surgery, The Fourth Hospital of Hebei Medical University, Shijiazhuang, Hebei 050011, P.R. China

Received September 23, 2011; Accepted November 17, 2011

DOI: $10.3892 /$ etm.2011.394

\begin{abstract}
T cell-mediated antitumor immunity is a cellular immune response that requires two signals. The dendritic cell (DC) has been considered as the most efficient antigen-presenting cell (APC). It plays essential roles in the induction, regulation and maintenance of antitumor immunity in humans. The 4-1BB/4-1BB ligand (4-1BBL) pathway plays crucial roles in immune response, tumor immunity and autoimmune diseases through transduction of $\mathrm{T}$ cell co-stimulatory signals. The aim of this study was to generate the preparation protocol for a DC vaccine transfected with gastric cancer cell total ribonucleic acid (RNA) carrying the 4-1 BBL gene in vitro and to investigate its antitumor effects in murine forestomach carcinoma (MFC). The vaccine was prepared by transfecting MFC total RNAs carrying the 4-1BBL gene into the DCs that were isolated from 615 mouse bones. The $\mathrm{T}$ cell proliferation rate in the MFC/4-1BBL/DC group was higher than that in the DC group. The tumor cell kill rate in the MFC/4-1BBL/DC group was higher than that in the DC group. ELISA analysis showed that IL-12 and IFN- $\gamma$ in the MFC/4-1BBL/DC group were more highly expressed compared to the other group. Collectively, our data demonstrate that the DC vaccine transfected with gastric cancer cell total RNA carrying the 4-1BBL gene has a stronger ability to kill gastric cancer cells through promoting $\mathrm{T}$ cell proliferation and enhancing the ability of cytotoxic T lymphocytes (CTLs) to kill gastric carcinoma cells and to secrete IL-12 and IFN- $\gamma$. Our results provide an effective therapeutic strategy for treating gastric cancer using a DC vaccine.
\end{abstract}

\section{Introduction}

Antitumor immunity mainly involves T cell-mediated cytoimmunity. T cell activation requires two signals. The first signal is specific. The second signal is non-specific and is provided by

Correspondence to: Dr Yong Li, Department of Surgery, the Fourth Hospital of Hebei Medical University, No. 12 Jiankang Road, Shijiazhuang, Hebei 050011, P.R. China

E-mail: songzhch@yahoo.com.cn

Key words: dendritic cell vaccine, 4-1BB ligand, gene transfection, murine forestomach carcinoma cell co-stimulatory molecules on the antigen presenting cell (APC) and corresponding ligands on the $\mathrm{T}$ cell. The absence of the second signal may lead to T cell anergy (1). The dendritic cells (DCs), as highly efficient and specialized APCs, are particularly crucial for the $\mathrm{T}$ cell-mediated immune response. The $4-1 \mathrm{BB} / 4-1 \mathrm{BBL}$ receptor/ligand pair in the immune system is another significant co-stimulatory signal other than CD28/B7, which plays an important role in immune response and tumor immunity, and also plays a part in cell adhesion, antigen presentation, T cell co-stimulation and signal transduction (2). In our study, we prepared a DC vaccine transfected with co-stimulatory molecule 4-1BBL transgenic murine gastric cancer cell total ribonucleic acid (RNA), in which the double signals may enhance the antitumor activity.

\section{Materials and methods}

Materials and reagents. A total of 615 mice (6-8 weeks old) were purchased from the laboratory animal center of the Chinese Academy of Medical Science (Tianjin, China). Mice were maintained in a specific-pathogen-free environment. All animal studies were approved by the ethics committee of the authors' institutional ethics committee. The murine forestomach carcinoma (MFC) cell strain was purchased from the Chinese Academy of Medical Science (Shanghai, China). The pMKITneo/4-1BBL plasmid was kindly provided by Professor Hideo Yagita. Lipofectamine 2000 was purchased from Invitrogen Corp. (Carlsbad, CA, USA). IL-12 and IFN- $\gamma$ ELISA kits were purchased from NeoBioscience Technology Company (Beijing, China).

MFC culture and 4-1BBL gene transfection. The DH5a bacterial strain containing the pMKITneo/4-1BBL plasmid was incubated in Lysogeny broth (LB). The plasmid DNA was extracted using a Wizard plus SV minipreps DNA purification system (Promega, Shanghai, China) and was digested by the restriction endonucleases $X h o \mathrm{I}$ and NotI, and then the identity of the plasmid was verified by electrophoresis on a $0.7 \%$ agarose gel.

MFC cell lines were cultured in RPMI-1640 supplemented with $10 \%$ fetal calf serum (FCS) in a humidified atmosphere containing $5 \% \mathrm{CO}_{2}$ at $37^{\circ} \mathrm{C}$.

The MFC cell line was transfected with the plasmid pMKITneo/4-1BBL using Lipofectamine 2000, and cultured in 
medium containing $0.4 \mathrm{~g} / 1 \mathrm{G} 418$ to screen 4-1BBL-expressing stable cell lines, MFC/4-1BBL.

RNA preparation and semi-quantitative reverse transcription polymerase chain reaction $(R T-P C R)$. Total mRNAs of the MFC and MFC/4-1BBL cell lines were purified using TRIzol (Invitrogen) according to the manufacturer's instructions. The clear $28 \mathrm{~S}$ and $18 \mathrm{~S}$ ribosomal RNA bands indicated a good quality of RNA. The total mRNA was subsequently amplified by RT-PCR. The PCR protocol included an initial denaturation step at $95^{\circ} \mathrm{C}$ for $5 \mathrm{~min}$, followed by 35 cycles with denaturation at $94^{\circ} \mathrm{C}$ for $45 \mathrm{sec}$, annealing at $56^{\circ} \mathrm{C}$ for $30 \mathrm{sec}$ and extension at $72^{\circ} \mathrm{C}$ for $45 \mathrm{sec}$. The primer used were as follows: forward, 5'-TCACTCGAGATGGACCAGCACACACTTGATG-3'; and reverse, 5'-GGCTGTTGGGTACCCTTACTCGCCGGCG AAT-3'. The length of the amplified product was $616 \mathrm{bp}$. $\beta$-actin was used as the internal control. The identity of the PCR products was verified by electrophoresis on a $1.5 \%$ agarose gel.

Generation of myeloid DCs. Myeloid DCs were isolated from the bone marrow of 615 mice as previously described (21). Briefly, the 615 mice were sacrificed and immersed in $75 \%$ ethanol for $2 \mathrm{~min}$. The femur and tibia were removed by aseptic surgery. The bones were then crushed and washed repeatedly with RPMI-1640 to remove bone marrow. The RPMI-1640 was centrifuged at $1,000 \mathrm{rpm}$ for $5 \mathrm{~min}$. Erythrocyte lysis buffer was added into the supernatant to remove red blood cells. The isolated myeloid DC cells were cultured in 6-well plates and maintained in the RPMI-1640 supplemented with $10 \%$ FCS, $10 \mathrm{ng} / \mathrm{ml} \mathrm{GM-CSF}$ and $10 \mathrm{ng} / \mathrm{ml}$ IL-4. The morphology of the cultured DCs, including shape and cell size, was carefully observed and verified by an inverted microscope on days 1, 3, 5 and 7 of isolation.

RNA transfection of DCs. The mouse DCs were cultured for 5 days. Then, $2 \mathrm{~h}$ prior to transfection, DCs were washed with RPMI-1640 serum-free medium 3 times and the cell density was adjusted to $1 \times 10^{6}$ cells/ml using RPMI-1640. For each transfection sample, the complex was prepared as follows: $10 \mu \mathrm{g}$ MFC/4-1BBL RNA were diluted in $500 \mu \mathrm{l}$ serum-free RPMI-1640, and $10 \mu$ l Lipofectamine 2000 were diluted in $500 \mu 1$ serum-free RPMI-1640. The complex was incubated for 5 min at room temperature. The diluted RNA was combined with diluted Lipofectamine 2000, mixed gently and incubated at $37^{\circ} \mathrm{C}$ in a $5 \% \mathrm{CO}_{2}$ incubator for $30 \mathrm{~min}$. The complex was added to the DC plates at $37^{\circ} \mathrm{C}$ in a $5 \% \mathrm{CO}_{2}$ incubator. The medium was changed after $4 \mathrm{~h}$, and the incubation of the DCs was continued.

ELISA assays were performed to measure IL-12 levels in the supernatant of immature DCs, DCs and MFC/4-1BBL/DCs.

The mRNAs of MFC/4-1BBL/DCs and DCs were purified using TRIzol, followed by amplification using RT-PCR. All products were analyzed by electrophoresis on $1.5 \%$ agarose gels.

Isolation and culture of mouse lymphocytes. The 615 mice were sacrificed. The spleen was removed on a 200-mesh sterilized copper net, and ground repeatedly. After washing with phosphate-buffered saline (PBS), single spleen cell suspension was collected and centrifuged. The pellet was mixed with PBS and mouse hydroxypropylmethyl cellulose at a 1:1 ratio, followed by centrifugation at 1,500 rpm for $15 \mathrm{~min}$. After washing 3 times with PBS, the isolated lymphocytes were cultured in the RPMI-1640 supplemented with 10\% FCS.

MTT lymphocyte proliferation. Mixed lymphocyte reactions were performed using mature DCs or MFC/4-1BBL/DCs as stimulator cells, and $\mathrm{T}$ lymphocytes as responder cells. Stimulator cells were incubated with $25 \mu \mathrm{g} / \mathrm{ml}$ mitomycin C (MMC) at $37^{\circ} \mathrm{C}$ for $30 \mathrm{~min}$ and cell density was adjusted to $4 \times 10^{4} / \mathrm{ml}, 2 \times 10^{4} / \mathrm{ml}$ or $1 \times 10^{4} / \mathrm{ml}$. Three repeats of each cell density were plated in 96-well plates.

The allogeneic lymphocytes at a density of $2 \times 10^{5} / \mathrm{ml}$ were used as responder cells. The lymphocytes were added into the stimulator cells. The responder cells or stimulator cells alone were used as the control. A total of $4 \mathrm{~h}$ prior to detection, MTT was added into the cells and incubated for $4 \mathrm{~h}$ in the dark, followed by addition of DMSO. The optical density (OD) at $570 \mathrm{~nm}$ was measured using the ELISA machine.

$\mathrm{T}$ cell proliferation rate $=[$ experimental OD $-($ responder cell OD + stimulator cell OD)]/responder cell OD x $100 \%$.

MTT tumor killing activity of DC-activated cytotoxic $T$ lymphocytes (CTLs). A tumor killing activity assay was performed using $\mathrm{T}$ cells together with mature DCs or MFC/4-1BBL/DCs as effector cells, and MFC cells as target cells. DCs or MFC/4-1BBL/DCs $\left(2 \times 10^{5} / \mathrm{ml}\right)$ were cultured in 12-well plates together with allogeneic lymphocytes $\left(2 \times 10^{5} / \mathrm{ml}\right)$ in the RPMI-1640 containing $10 \mathrm{ng} / \mathrm{ml}$ IL-2 on day 1,3 and 5 of culture. The stimulator to responder cells at the ratio of 1:20, 1:10 or 1:5 were mixed and added in 96-well plates. Three repeats of each cell density were performed. The stimulator cells or responder cells alone were used as the control. A total of $4 \mathrm{~h}$ prior to detection, MTT was added to the cells and incubated for $4 \mathrm{~h}$ in the dark, followed by addition of DMSO. The OD at $570 \mathrm{~nm}$ was measured by the ELISA machine.

Kill rate $=[$ experimental OD $-($ effector cells OD + target cells OD)]/effector cells OD x $100 \%$.

The IFN- $\gamma$ levels in the supernatant of the DCs or MFC/4-1BBL/DCs were measured using the ELISA kit.

Statistical analysis. All statistical calculations were carried out using SPSS 13.0. T-tests and ANOVA were used to compare the different groups. All values are indicated as the means \pm standard errors. $\mathrm{P}<0.05$ was considered to indicate a statistically significant difference.

\section{Results}

Verification of the pMKITneo/4-1BBL plasmid. The purified pMKITneo/4-1BBL plasmid was digested by XhoI and NotI. A 927 bp DNA fragment of the inserted 4-1BBL coding sequence was released from the $5.8 \mathrm{~kb}$ vector plasmid (Fig. 1). Our results indicate that the pMKITneo/4-1BBL plasmid was successfully purified.

Observation of DC morphology. The morphology of DCs was measured using an inverted microscope. On day 1, the majority of DCs were round or oval-shaped with a small size, and were attached well to the plate. On day 3 , the cell number was significantly increased with an enlarged cell size, and some 
Table I. The comparison of T cell proliferation in the different DC groups $(\mathrm{n}=3, \bar{x} \pm \mathrm{s})$.

\begin{tabular}{lccc}
\hline & \multicolumn{3}{c}{ Stimulation to responding ratio (\%) } \\
\cline { 2 - 4 } & $1: 20$ & $1: 10$ & $1: 5$ \\
\hline Group & & & \\
DC & $0.060 \pm 0.014$ & $0.148 \pm 0.017$ & $0.195 \pm 0.015$ \\
MFC/4-1BBL/DC & $0.093 \pm 0.012$ & $0.187 \pm 0.013$ & $0.240 \pm 0.021$ \\
P-value & 0.040 & 0.035 & 0.039 \\
\hline
\end{tabular}

DC, dendritic cell; MFC, murine forestomach carcinoma; 4-IBBL, 4-1BB ligand.

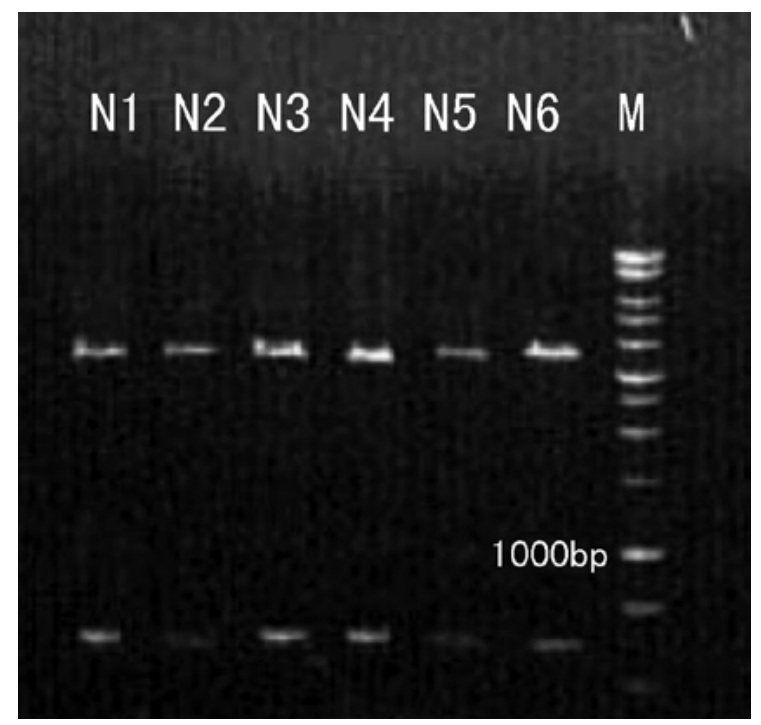

Figure 1. Verification of the pMKITneo/4-1BBL plasmid using the restriction endonuclease digestion. The release of a $927 \mathrm{bp} 4-1 \mathrm{BBL}$ DNA fragment and a $5.8 \mathrm{~kb}$ vector of the pMKITneo/4-1BBL plasmid by XhoI and NotI are shown. M, marker; N1-N6, pMKITneo/4-1BBL plasmid.

cells had grown as clustered colonies. On day 5, the number of cells was further increased and the cells were a similar size to that on day 3 . Some cells showed burr-like protuberance, and the cell colonies were markedly increased. On day 7 , the majority of the cells were detached from the plate with the nucleus deviated. A large number of burr-like protrusions were observed in characteristic irregular cells. Some cells contained round, star and dendritic-like protrusions. Notably, $12 \mathrm{~h}$ post-transfection, the morphology of cells returned to the state prior to transfection. Cells transfected with MFC total RNA carrying the 4-1BBL gene were round, and showed slightly reduced size, fewer surface protrusions and increased cell particles (Fig. 2).

MTT lymphocyte proliferation pre- and post-transfection. The ratio of stimulator cells to responder cells was 1:20, 1:10 or 1:5. The effects of the MFC/4-1BBL/DC group on T cell proliferation $(0.093 \pm 0.012,0.187 \pm 0.013,0.187 \pm 0.013)$ were significantly higher than that of the DC group $(0.060 \pm 0.014$, $0.148 \pm 0.017,0.195 \pm 0.015)(\mathrm{P}<0.05)$. We also noted that the
Table II. The activation of cytotoxic T lymphocyte (CTL) killing rate of tumor in the different DC groups $(n=3, \bar{x} \pm s)$.

\begin{tabular}{lccc}
\hline & \multicolumn{3}{c}{ Target/effector cell ratio (\%) } \\
\cline { 2 - 4 } & $1: 20$ & $1: 10$ & $1: 5$ \\
\hline Group & & & \\
DC+T cell & $0.342 \pm 0.023$ & $0.267 \pm 0.0243$ & $0.147 \pm 0.031$ \\
MFC/4-1BBL/ & $0.533 \pm 0.092$ & $0.321 \pm 0.018$ & $0.218 \pm 0.025$ \\
DC+T cell & & & \\
P-value & 0.025 & 0.036 & 0.037 \\
\hline
\end{tabular}

DC, dendritic cell; MFC, murine forestomach carcinoma; 4-IBBL, 4-1BB ligand.

higher stimulator cell to responder cell ratio appeared to show stronger T cell proliferation (Table I).

MTT tumor killing activity of DC-activated CTLs pre-and post-transfection. The ratio of target to effector cells was $1: 20,1: 10$ or 1:5. The effects of the MFC/4-1BBL/DC group tumor cell kill rate $(0.533 \pm 0.092,0.321 \pm 0.018,0.218 \pm 0.025)$ were higher than that of the DC group $(0.342 \pm 0.023$, $0.267 \pm 0.0243,0.147 \pm 0.031)(\mathrm{P}<0.05)$. Consistent with the proliferation results, higher target cell to effector cell ratio showed a stronger tumor cell killing rate (Table II).

The secretion of IL-12 and IFN- $\gamma$. We measured IL-12 and IFN- $\gamma$ secretion in the culture medium of different groups using ELISA analysis. We found that the IL-12 levels for the DC/ MFC/4-1BBL group were 20.240 $2.494 \mathrm{pg} / \mathrm{ml}, 17.088 \pm 3.933 \mathrm{pg} /$ $\mathrm{ml}$ for the DC group, and $10.288 \pm 2.390 \mathrm{pg} / \mathrm{ml}$ for the immature DC group $(\mathrm{P}<0.05)$. The IFN- $\gamma$ levels were $9.451 \pm 2.925 \mathrm{pg} / \mathrm{ml}$ for the DC/MFC/4-1BBL group and $5.979 \pm 1.639 \mathrm{pg} / \mathrm{ml}$ for the DC group $(\mathrm{P}<0.05)$.

\section{Discussion}

Gastric cancer is one of the most common malignant tumors. Tumor immune escape and tolerance are major problems in the treatment of the disease. Gene therapy provides a novel clinic strategy to enhance the immune function of tumor immunity. Upon activation by sensitized DCs, auxiliary T lymphocytes and CTLs are capable of killing tumors, which is the theoretical basis of tumor vaccines (2-4). There are a number of reports on DC vaccines in the treatment of melanoma, ovarian, colon (5), breast (6) and gastric cancer (7). Although some of them have been used in clinical antitumor treatment and have shown certain curative effects, we should have a better understanding of the patients, including human leukocyte antigen (HLA) phenotype and antigenic peptide epitopes. Recently, investigators have focused on DC vaccines loaded with total RNA with full tumor messages, and the advantages of this method are: no advance knowledge of the patient's HLA phenotype is required; total RNA contains multiple antigens and multiple epitopes of certain proteins that may be presented by different HLA types, therefore 


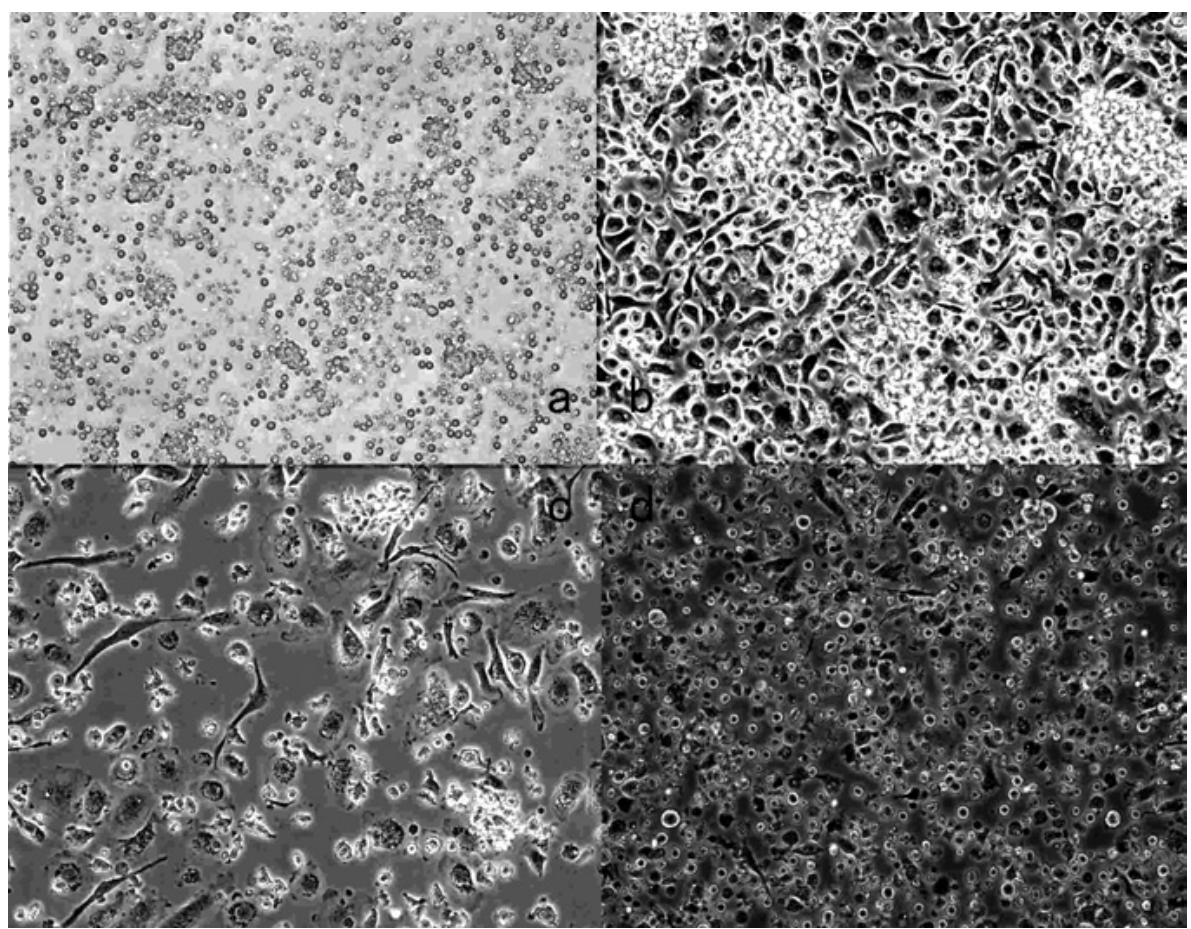

Figure 2. The morphology of DC and DC/4-1BBL in vitro. (a) On day 1, the majority of DCs were round, oval-shaped with a small size. (b) On day 5, the number of cells was further increased and some cells showed burr-like protuberance. (c) On day 7, the majority of the cells was detached from the plate with the nucleus deviated. Some cells contained round, star and dendritic-like protrusions. (d) Cells transfected with MFC total RNA carrying the 4-1BBL gene had a round, slightly reduced size, fewer surface protrusions and increased cell particles. DC, dendritic cell; MFC, murine forestomach carcinoma.

including a variety of tumor antigen encoded information; genetic material is easily available but not integrated into the host chromosome, so more secure; little damage to the DC is conductive to maintaining the activity and presenting function of the cell; RNA may be isolated from a small amount of tumor tissue and may be amplified to provide an adequate amount of material; comprehensive immune responses are induced to prevent antigenic variation and immune escape due to antigenic variation $(8-10)$.

The 4-1BB/4-1BBL receptor/ligand pair is another crucial co-stimulatory signal besides CD28/B7 in immune response and tumor immunity. It activates resting $\mathrm{T}$ cells separately or in coordination with $\mathrm{CD} 28$. It plays a significant role to maintain the existence and memory response of $\mathrm{T}$ lymphocytes (11). The secondary response of $\mathrm{CD}^{+} \mathrm{T}$ cells is highly dependent on 4-1BBL, which is also the case for some, though not all, secondary $\mathrm{CD}^{+} \mathrm{T}$ cell responses. Localized expression of immunostimulatory ligands using gene transfer approaches may reduce the risk of excessive, generalized immune activation observed in some clinical trials of systemically delivered antibodies $(12,13)$. Our investigation on DCs transfected with total RNA from murine gastric carcinoma cells with 4-1BBL gene in vitro, reduces the possibility of immune escape. It is a safe and easy method and has a potential clinical application $(9,12)$.

Studies using knockout mice have found that the secondary response of $\mathrm{CD}^{+} \mathrm{T}$ cells was highly dependent upon 4-1BBL. Though not all $\mathrm{CD} 4^{+} \mathrm{T}$ cells in the secondary response depended on the 4-1BBL, lasting tumor-specific $\mathrm{CD}^{+} \mathrm{T}$ cells may effectively lead to the response of CTL effector cells (14). 4-1BBL is an important co-stimulatory molecule in the
$\mathrm{T}$ cell immune response, which is capable of promoting the amplification of tumor-specific $\mathrm{T}$ cells $(14,15)$. The mature DCs transfected with 4-1BBL are capable of secreting large amounts of IL-12. It is known that IL-12 is one of the most important factors to promote helper $\mathrm{T}$ cell conversion to Th1 subsets and it is also a CTL differentiation factor $(16,17)$. The production of IL-12 is a critical step in the CTL activation process. It promotes CTL proliferation and generation of the protein perforin, induces the secretion of IFN- $\gamma$ and mediates the Th1 response. The 4-1BBL ligand also co-stimulates T cells and results in the secretion of IFN- $\gamma$ with CD28 (11), which is capable of promoting $\mathrm{T}$ cell differentiation to the Th1 type cells and preventing cytokine secretion in the Th2 cell (16).

Patients with malignant tumors can produce DCs with low expression of MHCI molecules, low secretion of IL-12 and reduced ability to uptake antigen and to stimulate $\mathrm{T}$ cells. Our results suggest that the effect on $\mathrm{T}$ cell proliferation in MFC/4-1BBL/DC groups was stronger than that in the DC group, and with the increased ratio of stimulator cells to responder cells, the $\mathrm{T}$ cell proliferation was enhanced, and the levels of IL-12 in the MFC/4-1BBL/DC group were significantly higher. Moreover, the tumor killing ability by CTLs activated with transfected DCs (MFC/4-1BBL/DC group) was stronger, and the levels of IFN- $\gamma$ of in the MFC/4-1BBL/DC groups were significantly higher than in other groups. 4-1BBL-transfected DCs may provide dual signals; therefore, their ability to promote $\mathrm{T}$ cell proliferation is significantly enhanced compared with non-transfected DCs, and that is induced to secrete IFN- $\gamma$, which directly activates $\mathrm{CD} 8^{+} \mathrm{T}$ cells and further enhances the specific tumor cell killing effect. 
The results of our study are consistent with ones from previous studies $(18,19)$. Therefore, the success of therapeutic vaccines will require formulations that are effective in: i) generating new immune responses, ii) boosting the existing immune responses, and iii) overcoming immune evasion mechanisms (20-22).

In conclusion, the results from our study suggest that the transfected DC vaccine enhances $\mathrm{T}$ lymphocyte proliferation and induces CTL cells to kill gastric cancer and promote the secretion of more IL-12 and IFN- $\gamma$. The results reported here provide the experimental basis for the clinical treatment of gastric cancer.

\section{Acknowledgements}

This study was supported by the Hebei Natural Sciences Foundation (No. C2008000966).

\section{References}

1. Lee SW, Park Y, So T, Kwon BS, Cheroutre H, Mittler RS and Croft M: Identification of regulatory functions for 4-1BB and 4-1BBL in myelopoiesis and the development of dendritic cells. Nat Immunol 9: 917-926, 2008.

2. Reis e Sousa C: Dendritic cells in a mature age. Nat Rev Immunol 6: 476-483, 2006.

3. Thomas-Kaskel AK and Veelken H: Active immunotherapy of prostate cancer with a focus on dendritic cells. Actas Urol Esp 31: 668-679, 2007.

4. Banchereau J and Palucka AK: Dendritic cells as therapeutic vaccines against cancer. Nat Rev Immunol 5: 296-306, 2005.

5. Yu JS, Liu G, Ying H, Yong WH, Black KL and Wheeler CJ: Vaccination with tumor lysate-pulsed dendritic cells elicits antigen-specific, cytotoxic T-cells in patients with malignant glioma. Cancer Res 64: 4973-4979, 2004.

6. Chan T, Sami A, El-Gayed A, Guo X and Xiang J: HER-2/ neu-gene engineered dendritic cell vaccine stimulates stronger HER-2/neu-specific immune responses compared to DNA vaccination. Gene Ther 13: 1391-1402, 2006.

7. Huarte E, Cubillos-Ruiz JR, Nesbeth YC, Scarlett UK, Martinez DG, Buckanovich RJ, Benencia F, Stan RV, Keler T, Sarobe P, Sentman CL and Conejo-Garcia JR: Depletion of dendritic cells delays ovarian cancer progression by boosting antitumor immunity. Cancer Res 68: 7676-7684, 2008.

8. Moyron-Quiroz JE, Rangel-Moreno J, Hartson L, Kusser K, Tighe MP, Klonowski KD, Lefrançois L, Cauley LS, Harmsen AG, Lund FE and Randall TD: Persistence and responsiveness of immunologic memory in the absence of secondary lymphoid organs. Immunity 25: 643-654, 2006.

9. Koski GK, Cohen PA, Roses RE, Xu S and Czerniecki BJ: Reengineering dendritic cell-based anti-cancer vaccines. Immunol Rev 222: 256-276, 2008.
10. Jordan KR, McMahan RH, Kemmler CB, Kappler JW and Slansky JE: Peptide vaccines prevent tumor growth by activating $\mathrm{T}$ cells that respond to native tumor antigens. Proc Natl Acad Sci USA 107: 4788-4789, 2010.

11. Krause P, Bruckner M, Uermösi C, Singer E, Groettrup M and Legler DF: Prostaglandin E(2) enhances T-cell proliferation by inducing the costimulatory molecules OX40L, CD70, and 4-1BBL on dendritic cells. Blood 113: 2451-2460, 2009.

12. Yi KH, Nechushtan H, Bowers WJ, Walker GR, Zhang Y, Pham DG, Podack ER, Federoff HJ, Tolba KA and Rosenblatt JD: Adoptively transferred tumor-specific T cells stimulated ex vivo using herpes simplex virus amplicons encoding 4-1BBL persist in the host and show antitumor activity in vivo. Cancer Res 67: 10027-10037, 2007.

13. Kuang Y, Weng X, Liu X, Zhu H, Chen Z, Jiang B and Chen H: Anti-tumor immune response induced by dendritic cells transduced with truncated PSMA IRES 4-1BBL recombinant adenoviruses. Cancer Lett 293: 254-262, 2010.

14. Shafer-Weaver KA, Watkins SK, Anderson MJ, Draper LJ, Malyguine A, Alvord WG, Greenberg NM and Hurwitz AA: Immunity to murine prostatic tumors: continuous provision of T-cell help prevents CD8 T-cell tolerance and activates tumor-infiltrating dendritic cells. Cancer Res 69: 6257-6264, 2009.

15. Habib-Agahi M, Phan TT and Searle PF: Co-stimulation with 4-1BB ligand allows extended T-cell proliferation, synergizes with CD80/CD86 and can reactivate anergic T cells. Int Immunol 19: 1383-1394, 2007.

16. Radhakrishnan S, Wiehagen KR, Pulko V, Van Keulen V, Faubion WA, Knutson KL and Pease LR: Induction of a Th1 response from Th2-polarized T cells by activated dendritic cells: dependence on TCR:peptide-MHC interaction, ICAM-1, IL-12, and IFN-gamma. J Immunol 178: 3583-3592, 2007.

17. Gautier EL, Huby T, Saint-Charles F, Ouzilleau B, Pirault J, Deswaerte V, Ginhoux F, Miller ER, Witztum JL, Chapman MJ and Lesnik P: Conventional dendritic cells at the crossroads between immunity and cholesterol homeostasis in atherosclerosis. Circulation 119: 2367-2375, 2009.

18. Drakes ML, Czinn SJ and Blanchard TG: Regulation of murine dendritic cell immune responses by Helicobacter felis antigen. Infect Immun 74: 4624-4633, 2006.

19. Jenkins MR, Mintern J, La Gruta NL, Kedzierska K, Doherty PC and Turner SJ: Cell cycle-related a quisition of cytotoxic mediators defines the progressive differentiation to effector status for virus specific CD8 ${ }^{+}$T cells. J Immunol 181: 3818-3822, 2008.

20. Sharma RK, Elpek KG, Yolcu ES, Schabowsky RH, Zhao H, Bandura-Morgan L and Shirwan H: Costimulation as a platform for the development of vaccines: a peptide-based vaccine containing a novel form of 4-1BB ligand eradicates established tumors. Cancer Res 69: 4319-4326, 2009.

21. Kuroda T, Kitadai Y, Tanaka S, Yang X, Mukaida N, Yoshihara M and Chayama K: Monocyte chemoattractant protein-1 transfection induces angiogenesis and tumorigenesis of gastric carcinoma in nude mice via macrophage recruitment. Clin Cancer Res 11: 7629-7636, 2005.

22. Xie SJ, Yan QH, Shan BE, Fu ZX, Meng FJ, Li BD and Cai JH: In vitro experimental study individualised immunotherapy induced by dendritic cells transfected with total RNA of autologous gastric cancer cells. Xi Bao Yu Fen Zi Mian Yi Xue Za Zhi 22: 92-95, 2006. 